\title{
Pyoderma gangrenosum near a cystostomy catheter
}

\author{
Michael Arthur Santos ${ }^{1,2} \cdot$ Reza Manesh $^{3} \cdot{\text { Tonya } \text { Crook }^{4}}^{4}$
}

Received: 18 July 2017/ Accepted: 24 July 2017/Published online: 29 July 2017

(C) SIMI 2017

A 78-year-old man with chronic lymphocytic leukemia and neurogenic bladder with an indwelling suprapubic cystostomy presented with a small, painful lump surrounding his catheter site. The throbbing, lancinating pain started after a routine outpatient catheter exchange at his urologist's office. He reported that the pustule spread in a centrifugal fashion, and degenerated into an ulcer. He was prescribed cephalexin for presumed cellulitis by his primary care doctor.

In the ensuing $72 \mathrm{~h}$, the lesion continued to expand and was accompanied with increased pain despite taking antibiotics. He reported subjective fevers and night sweats, and subsequently presented to an outside hospital, where he was hospitalized for 5 days to receive intravenous antibiotics for presumed failure of oral therapy. He received vancomycin and piperacillin-tazobactam empirically. His blood cultures were negative for bacteria and fungi, and because of a mild improvement in symptoms, he was discharged home with amoxicillin-clavulanic acid to complete a 10-day course. His wound did not improve. He re-presented 5 days after discharge with worsening, uncontrolled pain and a continuously sprawling ulcer.

Michael Arthur Santos

msantos1@ pennstatehealth.psu.edu

1 Department of Medicine, Penn State Milton S Hershey Medical Center, Hershey, USA

2 Department of Medicine, WellSpan Good Samaritan Hospital, Lebanon, PA, USA

3 Department of Medicine, Johns Hopkins University, Baltimore, MD, USA

4 Department of Infectious Diseases, Penn State Milton S Hershey Medical Center, Hershey, USA
Vital signs upon presentation showed a temperature of $38.4{ }^{\circ} \mathrm{C}$, heart rate of 121 beats $/ \mathrm{min}$, blood pressure of $144 / 81 \mathrm{mmHg}$, and respiration rate of 18 breaths $/ \mathrm{min}$. Abdominal examination revealed normal bowel sounds throughout, no tenderness to palpation away from the ulcer site, non-tender splenomegaly, and no rebound tenderness or guarding. Skin examination on the lower abdomen demonstrated a $30 \times 10 \mathrm{~cm}$ ulcer with gunmetal gray, undermined borders (Fig. 1). He had exquisite tenderness to light touch over the ulcer. Laboratory studies showed a complete blood count consistent with a known pancytopenia from chemotherapy received 4 months previously, noting that his white blood cell count of $3270 / \mu \mathrm{L}$ (ANC $2808 / \mu \mathrm{L}$ ), hemoglobin $8.8 \mathrm{~g} / \mathrm{dL}$, and platelet count of $100,000 / \mu \mathrm{L}$ were relatively unchanged. A peripheral blood smear was consistent with chemotherapy-induced pancytopenia. Urinalysis, liver function, and coagulation studies were within normal range. Urine and serum antigen studies for Histoplasma capsulatum and Blastomyces dermatitidis were negative. Repeat blood cultures were negative for fungal elements and bacteria. Abdominal computed tomography scan showed superficial abdominal wall stranding, but no signs of abscess. A biopsy of the ulcer edge showed a diffuse dermal infiltrate composed of mature neutrophils. Gram stains and special stains for microorganisms (Periodic Acid Schiff, Gomori Methenamine Silver, and Acid-Fast Bacilli) were negative for any bacteria and subsequent tissue culture yielded no growth. The biopsy site also was exquisitely painful and started to spread. His painful lesion progressed despite treatment with vancomycin and meropenem for 5 days and his pain was inadequately controlled despite multiple analgesics.

Given his failure to improve with broad spectrum antibiotics, the onset of his illness was revisited. He stressed that the wound worsened immediately after his 


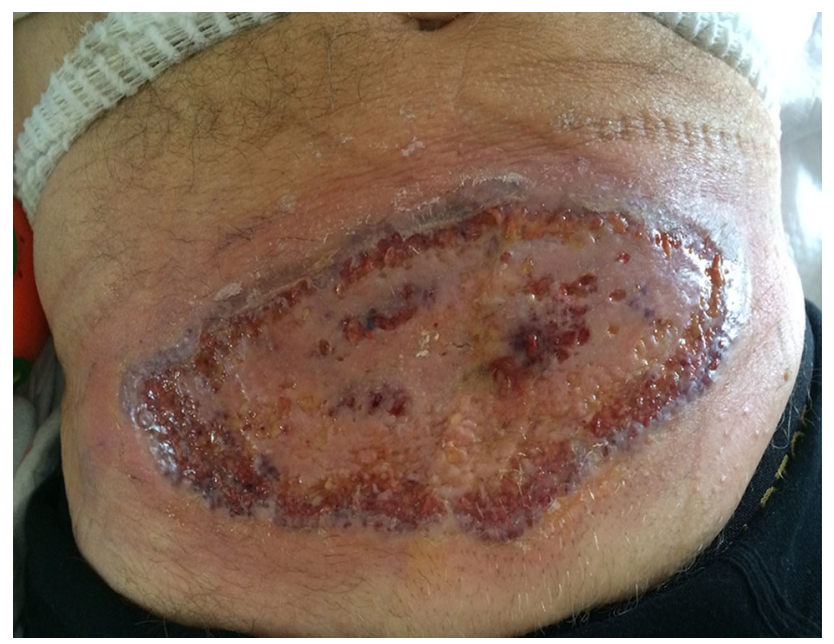

Fig. 1 Lower abdominal skin lesion image 2 weeks after its initial onset, showing a large oval-shaped lesion with sharp, ulcerated borders, and gray overhanging borders. The suprapubic catheter has been removed

cystostomy exchange. Due to a negative infectious workup, the characteristics of a sprawling ulceration after trauma, and sterile neutrophilic infiltrate on skin biopsy, he was diagnosed with pyoderma gangrenosum (PG). The antibiotics were discontinued, and he was started on high dose intravenous methylprednisolone. Within $24 \mathrm{~h}$, his pain rapidly improved. He was discharged 2 days later on a prednisone taper, and his wound healed over a course of 3 weeks. He tolerated reinsertion of his suprapubic catheter upon outpatient follow-up without complications, and experienced mild pathergy at the biopsy site, which resolved with triamcinolone injections.

First described more than 80 years ago, PG remains a vague and challenging diagnosis. It is a painful, inflammatory skin condition that falls on the continuum of inflammatory neutrophilic dermatoses, such as Sweet's syndrome, subcorneal pustular dermatosis, dermatitis herpetiformis, and Behcet's disease, amongst many others. PG can present on a broad clinical spectrum, masquerading as non-healing ulcers, necrotizing fasciitis, and septic shock. The differential diagnosis is broad; therefore, careful investigation of both non-infectious skin lesions, such as vasculitis, cutaneous malignancies, autoimmune disorders, and infectious etiologies (ecthyma, fungal, and mycobacterial) should be performed. Half of all cases are idiopathic, while the remainder occurs in the setting of inflammatory bowel disease (IBD), hematologic malignancy, seronegative spondyloarthritis, and connective tissue disease [1]. The pathophysiology of PG is poorly understood, and is believed to be secondary to underlying neutrophil and monocyte dysfunction.
Anatomically, PG most commonly occurs on the lower extremities, though nearly any site can be affected, including near sites of mild trauma. Non-dermatologic manifestations have been described, including ocular, pulmonary, splenic, cardiac, and joint involvement [1]. Pathergy, or exacerbation of a wound following trauma, is a key feature in PG, and is familiar to some clinicians more for its association with Behçet's disease [2-4].

PG is described as four clinical variants: ulcerative, bullous, pustular, and vegetative, distinguished by historical features and histology. Ulcerative PG often presents in the setting of an underlying malignancy, rheumatoid arthritis or IBD. Bullous PG is associated with myeloproliferative disorders, such as acute leukemia and myelodysplastic syndrome. Ulcerative and peristomal forms of PG are seen with IBD, sacroiliitis, monoclonal gammopathy, and malignancy. Vegetative PG is a less aggressive form without any clear disease association. Our patient's presentation was consistent with ulcerative PG given the historical onset of the lesion and the dense neutrophilic infiltrate on histology. Chronic lymphocytic leukemia is not a commonly associated underlying malignancy and, therefore, it was not believed to be clinically significant.

PG is a diagnosis of exclusion. There are no diagnostic serologies or pathognomonic histology findings, and while biopsy is not necessary for diagnosis, tissue sampling is useful for ruling out infection or malignancy. Clinical criteria have been proposed, but none have been universally accepted, adding to the quandary that many clinicians face when trying to diagnose PG. The criteria used in this case were the historical component of pathergy, ulcer expansion of 1-2 cm per day, clinical improvement in response to steroids, and sterile histologic dermal neutrophilia [1]. In this case, revisiting the history told the typical tale of PG: history of minor trauma followed by a painful, rapidly expanding wound that failed to respond to antibiotics.

Currently, there are no gold standard treatments for PG. First-line pharmacological therapy begins with an empiric trial of combination oral and topical steroids or cyclosporine. The response is generally favorable, measured by pain relief and slowed expansion of the lesion [5]. The use of other agents has been reported, including topical or systemic immunosuppression with mycophenolate mofetil, methotrexate, azathioprine, and anti-tumor necrosis factor alpha inhibitors in refractory cases. Wound care is a vital component to management for preventing super-infection. Lesions are susceptible to repeat trauma, therefore, consultation with a dermatologist and a wound care specialist is warranted. 
Unfortunately, PG was not considered early in the course, and this led to an excessive use of broad spectrum antibiotics because of premature closure on the diagnosis of cellulitis. Antibiotics were indicated initially; however, the failure to respond clinically should lead clinicians to revisit other diagnostic possibilities. Recognition of this condition in particular is crucial because it can influence the decision to discontinue antibiotics and initiate appropriate immunosuppressive therapy in a timely manner, sparing the patient any further discomfort.

Acknowledgements The authors would like to thank Dr. Catherine Chung, MD and Dr. Young Lee, MD for their initial review of the manuscript.

\section{Compliance with ethical standards}

Conflict of interest The authors declare that they have no conflict of interest.

Statement of human and animal rights This article does not contain any studies with human or animal subjects performed by any of the authors.
Informed consent Informed consent was obtained from the individual in this report prior to submission of this manuscript.

Funding There are no funding sources to report.

\section{References}

1. Ahronowitz I, Harp J, Shinkai K (2012) Etiology and management of pyoderma gangrenosum-a comprehensive review. Am J Clin Dermatol 13(3):191-211

2. Alavi A, Sajic D, Cerci FB, Ghazarian D, Rosenbach M, Jorizzo J (2014) Neutrophilic dermatoses: an update. Am J Clin Dermatol 15(5):413-423

3. Su WP, Davis MD, Weenig RH, Powell FC, Perry HO (2004) Pyoderma gangrenosum: clinicopathologic correlation and proposed diagnostic criteria. Int J Dermatol 43(11):790-800

4. Varol A, Seifert O, Anderson CD (2010) The skin pathergy test: innately useful? Arch Dermatol Res 302(3):155-168

5. Reichrath J, Bens G, Bonowitz A, Tilgen W (2005) Treatment recommendations for pyoderma gangrenosum: an evidence-based review of the literature based on more than 350 patients. J Am Acad Dermatol 53(2):273-283 